\title{
Simulation Results for a Crosstalk Avoidance and Low Power Coding Scheme for System on Chips
}

\author{
Anitta Thomas and Shinoj J Vattakuzhi
}

\begin{abstract}
The major problems associated with Systemon-Chip buses are Delay problem, Power problem and Reliability problem. Capacitive crosstalk and high power consumption due to various capacitances are the major causes of this problem. So, inorder to avoid these problems, a coding scheme is proposed which avoids crosstalk, reduces power consumption and increases reliability. This involves design of encoder and decoder modules. VHDL simulation of encoder and decoder modules are done using GHDL simulator.
\end{abstract}

Keywords--- Bus-invert, Crosstalk, Correlated Switching, Forbidden Pattern

\section{INTRODUCTION}

$\mathrm{S}$ YSTEM-ON-CHIP is the technology by which different systems that perform various functions are placed on a single silicon chip. This inturn reduces the development cycle, increase product functionality, performance and quality. A system-on-chip is designed to provide full functionality for an application by stitching together multiple stand-alone VLSI designs (cores). The major advantages achieved by SoC devices are lower cost, decreased size and reduced power consumption. System-on-Chips are widely used in embedded systems.

A significant amount of the power dissipated in digital circuits is due to long buses. With the Deep Sub Micron (DSM) technology in CMOS, the interconnect width and spacing reduce, which in turn increases the inter-wire capacitance and interconnect resistance. Such shrinking feature size, makes the power dissipation in data bus more predominant than the power dissipation in other parts of the circuitry.

As the number of multiple function systems in a single chip increases, on-chip global interconnects length are also increasing. This results in large on-chip propagation delay due to increase in interconnect resistance, length, and inter-wire capacitance. The wire delay is becoming a large fraction of the overall delay of a circuit[1]. The wire delay contributes to the major fraction of overall delay.

Anitta Thomas, Assistant Professor, Department of Electronics \& Communication Engineering, Viswajyothi College of Engineering and Technology, Vazhakulam, Ernakulam, Kerala, India. E-mail:anitta.t@gmail.com

Shinoj J Vattakuzhi, Sub Divisional Engineer, Bharath Sanchar Nigam Ltd, Ernakulam, Kerala, India.E-mail:shinojvattakuzhi@gmail.com DOI: 10.9756/BIJRCE.10444
The system block diagram is given in Figure 1:

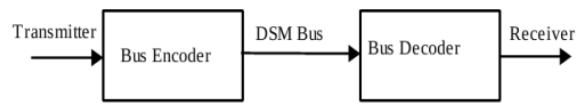

Figure 1: System Block Diagram

The system is designed in such a way that it overcomes problems mentioned above.

\section{LITERATURE SURVEY}

Crosstalk is caused by the interference of one signal with another signal. On chip crosstalk is mainly due to the capacitive coupling of nearby lines. The capacitive crosstalk increases when two adjacent lines are switching simultaneously in opposite direction. Crosstalk in the adjacent wires causes timing violations and extra power dissipation [3].

Two problems [2] can occur due to cross-talk. First, there is a significant delay variation may occur in the victim wire depending on the state of neighboring wires due to crosstalk. Second, loss of signal integrity may occur due to the misinterpretation of the logical state of the wire due to crosstalk of aggressor wire. Chances of correlated switching is also there when two or more neighboring wires make opposite transitions at the same time. For example, the victim wire is switching from high to low while its adjacent wire (aggressor) is switching from low to high at the same time.

Different possibilities of signal transitions in a data bus is analyzed in Figure 2. In type A transitions (Figure 2 (a)), there is no correlated switching and the cross-coupling capacitance is minimum. In type B transitions (Figure 2 (b)), there is one correlated switching. In this case, the effect of coupling capacitance between line 1 and line 2 increases twice due to Miller effect. The type C transition (Figure 2(c)) is the worst possible scenario where there are two correlated switchings between the wire and its two adjacent neighbors. Here the coupling capacitance between both adjacent neighboring wires increases by a factor of 2 due to Miller effect.

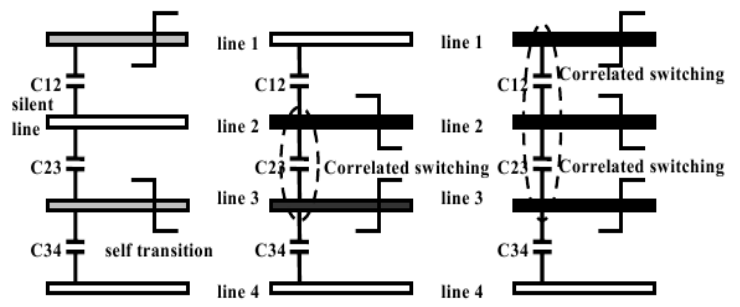
(a) Type A
(b) Type B
(c) Type C

Figure 2: Categorization of Signal Transitions in a bus 


\section{A. Terminologies related to Crosstalk}

The coupling capacitance of a wire can be classified into four types $1 \mathrm{C}, 2 \mathrm{C}, 3 \mathrm{C}$ and $4 \mathrm{C}$ [4] according to the coupling capacitance (CI) of two wires. Let (vi, vi+1, vi+2) represent the signal pattern on three wires at any given time, where $v i+1$ is the victim, and vi,$v i+2$ are the aggressors. $j$ and $j+1$ represents two instants of time.

1.C sequence: A sequence of vectors is called a 1.C sequence [1] if

$$
\begin{aligned}
& \text { - } v_{i}^{V j}=v_{i}^{V j+1}=01 \text { and } v_{i+1}{ }^{V j}=v_{i+2}{ }^{V j}=v 2 \text { and }
\end{aligned}
$$

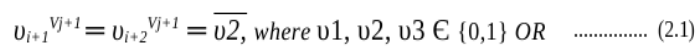

$$
\begin{aligned}
& \text { - } v_{i+2}{ }^{V_{j}}=v_{i+2}{ }^{{ }^{j}+1}=01 \text { and } v_{i}^{V_{j}}=v_{i+1}{ }^{V_{j}}=02 \text { and } \\
& v_{i}^{V j+1}=v_{i+1}{ }^{V+1}=\overline{v 2} \text {, where v1, v2, u3 } €\{0,1\} .
\end{aligned}
$$

The coupling capacitance for 1.C sequence is CI [4].

2.C sequence: A sequence of vectors is called a $2 \mathrm{C}$ sequence if

$$
\begin{aligned}
& v_{i}^{V j}=v_{i}^{V j+1}=01 \text { and } v_{i+1}^{V j}=02 \text { and } v_{i+1}^{V j+1}=\overline{v 2} \\
& \text { and } v_{i+2}{ }^{V_{j}}=v_{i+2}^{V_{j}+1}=03 \text { where } \mathrm{v1}, \mathrm{v} 2, \mathrm{v} 3 €\{0,1\} \text {. }
\end{aligned}
$$

The coupling capacitance for 2.C sequence is $2 \mathrm{CI}$.

3.C sequence: A sequence of vectors is called a 3C sequence if

$$
\begin{aligned}
& \text { - } v_{i}^{V j}=v_{i+1}^{V+1}=v 1 \text { and } v_{i}^{V j+1}=v_{i+1}^{V j}=\overline{v 1} \\
& \text { and } v_{i+2}{ }^{V j}=v_{i+2}{ }^{V j+1}=02 \text { where } 01,02 €\{0,1\} O R \\
& \text { - } v_{i+1}{ }^{V_{j}}=v_{i+2}{ }^{V_{j+1}}=01 \text { and } v_{i+1}^{{ }^{j j+1}}=v_{i+2}{ }^{V_{j}}=\overline{v 1} \\
& \text { and } u_{i}^{V j}=v_{i}^{V j+1}=v 2 \text { where } v 1, v 2 €\{0,1\} \text {. }
\end{aligned}
$$

The coupling capacitance of $\boldsymbol{v}_{i+1}$ in this case will be 3CI.

4.C sequence: A sequence of vectors is called a 4C sequence if

The coupling capacitance of $\boldsymbol{v}_{\boldsymbol{i}+1}$ in this case rises to $4 \mathrm{CI}$.

\section{B. Delay Model for the DSM Bus}

The delay of line $1(1<1<\mathrm{n})$ of the bus is given by [5]

$$
v_{i}^{V j}=v_{i+1}^{V+1}=v_{i+2}^{V j}=v \text { and } v_{i}^{V j+1}=v_{i+1}^{V y}=v_{i+2}{ }^{V+1}=\bar{v},
$$

where $v \in\{0,1\}$

$$
T_{l=\tau_{0}}\left[(1+2 \lambda) \Delta_{l}^{2}-\lambda \Delta_{l}\left(\Delta_{l-1}+\Delta_{l+1}\right)\right]
$$

$\tau_{0}$ - delay of a crosstalk-free line

$\lambda$ - ratio of the coupling capacitance to the bulk capacitance $\Delta \mathrm{l}$ - is the transition occurring on line 1 .
$\Delta \mathrm{l}$ is equal to 1 for 0 -to- 1 transition, -1 for 1 -to- 0 transition, and 0 for no transition. The relative delay of a line 1 is given in Table 1.

Table 1: Relative Delay of Line 1

\begin{tabular}{|c|c|c||c|}
\hline$\Delta_{l-1}$ & $\Delta_{l}$ & $\Delta_{l+1}$ & Relative Delay \\
\hline- & - & - & 0 \\
\hline$\uparrow$ & $\uparrow$ & $\uparrow$ & 1 \\
\hline- & $\uparrow$ & $\uparrow$ & $1+\lambda$ \\
\hline- & $\uparrow$ & - & $1+2 \lambda$ \\
\hline$\uparrow$ & $\uparrow$ & $\downarrow$ & $1+2 \lambda$ \\
\hline$\downarrow$ & $\uparrow$ & - & $1+3 \lambda$ \\
\hline$\downarrow$ & $\uparrow$ & $\downarrow$ & $1+4 \lambda$ \\
\hline
\end{tabular}

\section{Crosstalk Avoidance Coding AND Decoding}

\section{A. Forbidden Pattern Based Crosstalk Avoidance Coding}

The patterns "101" and "010" are forbidden patterns. If there is no forbidden pattern in any three consecutive bits, then the code is said to be forbidden pattern free (FPF). As an example, 1101110 is not forbidden pattern free; 1100110 is FPF.

Table 2 shows a particular mapping which eliminates 3C (and 4C) crosstalk sequences [1], and also eliminates 010 and 101 patterns.

For the particular encoding shown in Table 2, the logic

functions are:

$$
\begin{aligned}
& \mathrm{q} 1=\mathrm{d} 1 \\
& \mathrm{q} 2=(\mathrm{d} 2 \mathrm{~d} 3+\mathrm{d} 2 \overline{\mathrm{d} 4}) \oplus \mathrm{d} 1 \cdots \cdots \cdots \cdots \cdots \cdots \cdots \cdot(2.8) \\
& \mathrm{q} 3=(\mathrm{d} 2+\mathrm{d} 3 \overline{\mathrm{d}} 4) \oplus \mathrm{d} 1 \\
& \mathrm{q} 4=(\mathrm{d} 3+\mathrm{d} 2 \mathrm{~d} 4) \oplus \mathrm{d} 1 \quad \ldots \ldots \ldots \ldots \ldots \ldots \ldots(2.10) \\
& \mathrm{q} 5=\mathrm{d} 4 \oplus \mathrm{d} 1
\end{aligned}
$$

If a 16-bit bus is considered, the input is divided into four 4-bit groups. The data of each group is encoded using a 4 to 5 encoder. For a 4-bit bus, all $2^{4}=16$ vectors can be mapped to 16 available 5-bit vectors as shown in Table 2. In practice, forbidden pattern can occur across the group boundaries. To prevent this from occurring, the outputs of the second encoder is inverted if $\mathrm{v}_{\mathrm{j}, 5} \neq \mathrm{v}_{\mathrm{j}+1,1}$ where $j$ is the group index. To identify this at the decoder end, a group complement bit is also transmitted. The complete set of grouping complement bits are transmitted in a separate group. 
Table 2: 4 to 5 Encoder Input-Output Mapping

\begin{tabular}{|l|l|}
\hline Input & output \\
\hline \hline 0000 & 00000 \\
\hline 0001 & 00001 \\
\hline 0010 & 00110 \\
\hline 0011 & 00011 \\
\hline 0100 & 01100 \\
\hline 0101 & 00111 \\
\hline 0110 & 01110 \\
\hline 0111 & 01111 \\
\hline 1000 & 11111 \\
\hline 1001 & 11110 \\
\hline 1010 & 11001 \\
\hline 1011 & 11100 \\
\hline 1100 & 10011 \\
\hline 1101 & 11000 \\
\hline 1110 & 10001 \\
\hline 1111 & 10000 \\
\hline
\end{tabular}

The block diagram of 16-bit Crosstalk Avoidance Coding is shown in Figure 3.

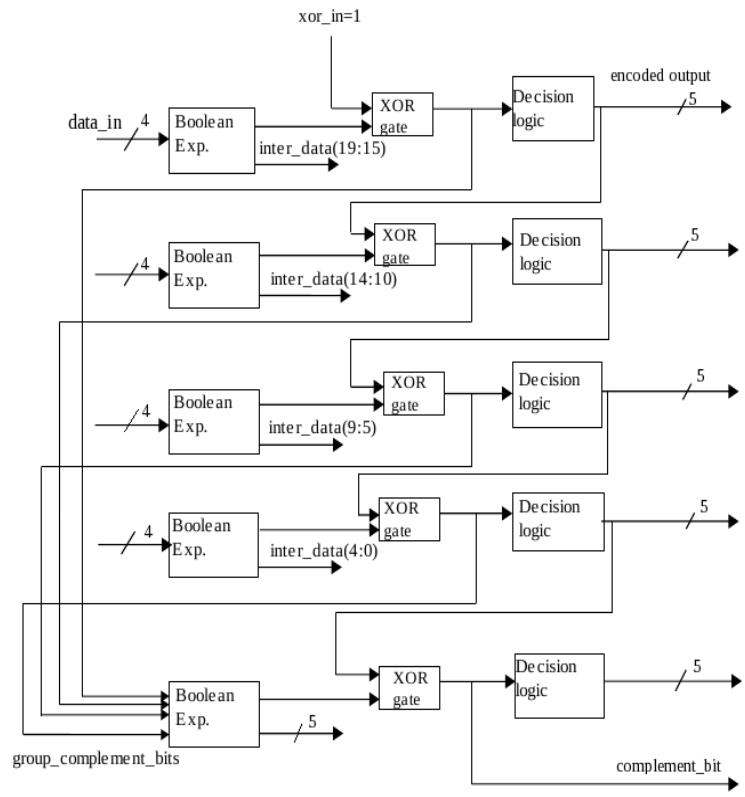

Figure 3: Block Diagram for Crosstalk Avoidance Coding

As in Figure, each group is coded using the boolean expressions discussed (Equations. 2.7 to 2.11). The inversion of inter_data values is determined by decision logic according to XOR gate output. The final encoded value of a particular group is compared with the boundary bits of the next group and so on. The advantage of xor_in input is that any number of 16-bit data busses can be cascaded to form higher bus width. The xor_in can be complement_bit from other group of 16-bit data.

\section{B. Advantages of Crosstalk Avoidance codes}

- Crosstalk Avoidance Codes reduces the mutual interwire coupling capacitance and hence the energy dissipation of wire segments.

- Reduces the inter-wire crosstalk and hence boosts up the maximum speed on the data bus.

- System reliability can be enhanced and overall energy dissipation can be decreased.

- Less area overhead than shielding techniques [8].

\section{Crosstalk Avoidance Decoding}

Table 3 shows a 5 to 4 decoder input-output mapping which decodes the data pattern coded using the method discussed in section $\mathrm{C}$.

Table 3: 5 to 4 Decoder Input-Output Mapping

\begin{tabular}{|l|l|}
\hline Input & output \\
\hline \hline 00000 & 0000 \\
\hline 00001 & 0001 \\
\hline 00110 & 0010 \\
\hline 00011 & 0011 \\
\hline 01100 & 0100 \\
\hline 00111 & 0101 \\
\hline 01110 & 0110 \\
\hline 01111 & 0111 \\
\hline 11111 & 1000 \\
\hline 11110 & 1001 \\
\hline 11001 & 1010 \\
\hline 11100 & 1011 \\
\hline 10011 & 1100 \\
\hline 11000 & 1101 \\
\hline 10001 & 1110 \\
\hline 10000 & 1111 \\
\hline
\end{tabular}

For the particular decoding shown in Table 3, the logic functions are:

$$
\begin{aligned}
& \mathrm{d} 1=\mathrm{q} 1 \\
& \mathrm{~d} 2=(\mathrm{q} 1 \oplus \mathrm{q} 2)+\overline{\mathrm{q} 1} \mathrm{q} 3 \mathrm{q} 5+\mathrm{q} 2 \overline{\mathrm{q} 3} \overline{\mathrm{q} 5} \\
& \mathrm{~d} 3=\overline{\mathrm{q} 1}(\mathrm{q} 2 \mathrm{q} 4+\overline{\mathrm{q} 3} \mathrm{q} 4+\mathrm{q} 4 \overline{\mathrm{q}})+\mathrm{q} 1(\overline{\mathrm{q} 2} \overline{\mathrm{q} 4}+\mathrm{q} 3 \overline{\mathrm{q} 4}+\overline{\mathrm{q} 4} \mathrm{q} 5) \\
& \mathrm{d} 4=\mathrm{q} 1 \oplus \mathrm{q} 5
\end{aligned}
$$

The block diagram for 16-bit Crosstalk Avoidance Decoding is given in Figure 4.

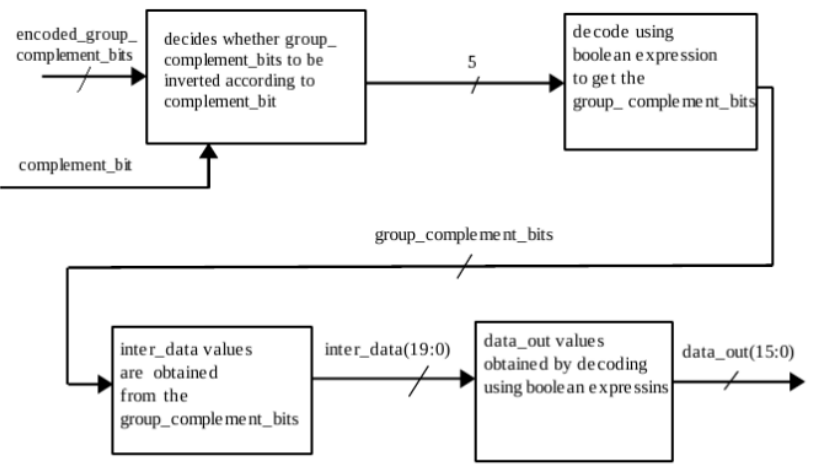

Figure 4: Block Diagram for Crosstalk Avoidance Decoding 
The input complement_bit represents whether the final encoded value of group complement bits is inverted or not. The group complement bits are decoded using boolean expressions(Equations 2.12 to 2.15). The data_out values were obtained by using group complement bits and also by decoding using the boolean expressions discussed in this section. The data_out values are same as that of the data given to the crosstalk avoidance coding block.

\section{LOW POWER CODING AND DECODING}

Bus Invert Coding is the method applied for obtaining low power. Low power encoding techniques transform the data in such a way that the self-switching activity on buses is reduced.

\section{A. Bus-Invert Coding}

In the bus-invert method [6,7], the Hamming distance is computed between the present bus value and the next data value (also counting the present invert line). If the Hamming distance is larger than $\mathrm{n} / 2$, set 'invert' is equal to ' 1 ' and make the next bus value equal to the inverted next data value. Otherwise let 'invert' is equal to ' 0 ' and let the next bus value equal to the next data value. At the receiver side the contents of the bus must be conditionally inverted according to the invert line.

The block diagram for bus invert coding is shown in Figure 5. The XOR gate plays a major role in calculating the hamming distance. If the output of XOR logic is one, it means that the corresponding bits of the present data value and the previous bus value differ. The majority voter circuit takes the decision whether to invert the next bus value according to the Hamming distance. The value of the present invert line must be considered by the majority voter. The decision logic outputs the bus value according to the input obtained from the majority voter circuit.

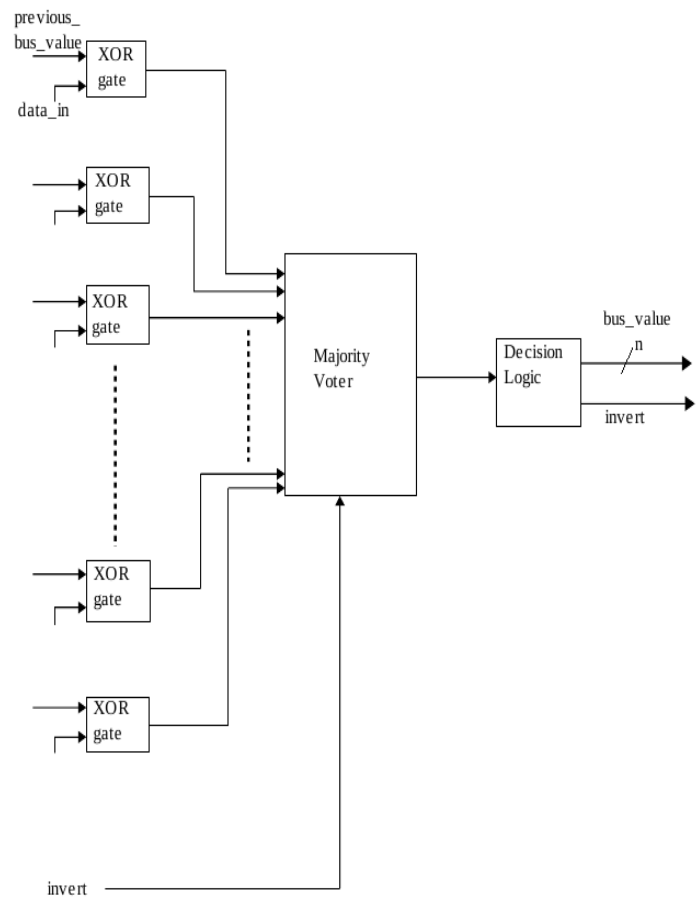

Figure 5: Block Diagram for Bus Invert Coding

\section{B. Bus-Invert Decoding}

The block diagram for bus invert decoding is given in Figure 6. The invert bit carries the information about the inversion of data value. The decision logic is the logic which decides whether the input data to this block to be inverted to get the original data value.

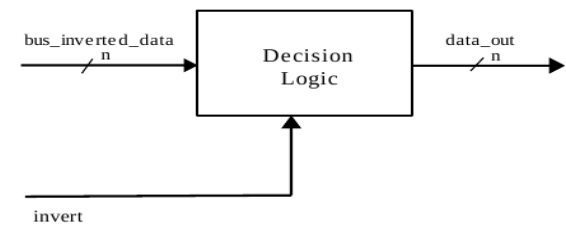

Figure 6: Block Diagram for Bus Invert Decoding

\section{IMPLEMENTATION}

In the proposed coding scheme to address the cross talk and to reduce the power consumption, it was decided to place FPC (for crosstalk avoidance) as the first block to ensure that after performing bus invert coding(for low power) also, the crosstalk avoidance property will be maintained. If the coding scheme is reversed, that is first bus invert coding followed by crosstalk avoidance coding; then the criteria for low power consumption will not be met. The low-power, reliable, crosstalk avoidance coding scheme had been implemented for 8 bits of data as well as for 16 bits of data. The 8-bit data coding scheme has the disadvantage that, it cannot be used for higher bus widths.So, a 16-bit data coding scheme is proposed which can be extended to a higher bus width of 32 bits, 48 bits, 64 bits etc. This can be used as a core in future systemon-chips.

\section{A. Encoder Module}

The block diagram for the encoder module is shown in Figure 7. The CAC block and the bus invert coding block were integrated to get the encoder module. The first block eliminates crosstalk and the second block is designed for low power. The input to this integrated block is data_in(15:0) and the output obtained is bus_out(25:0) and invert bit, the coded value given to the bus.

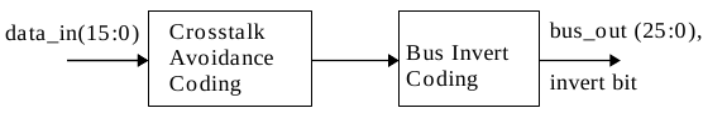

Figure 7: Block Diagram for 16-bit Encoder Module

\section{B. Decoder Module}

The block diagram for the encoder module is shown in Figure 8 . The bus invert decoding block and the crosstalk avoidance decoding block were integrated to get the decoder module. The input to this integrated block is the output obtained from encoder module. The output of decoder module is denoted as data_out(15:0), which is same as the data given to the encoder module.

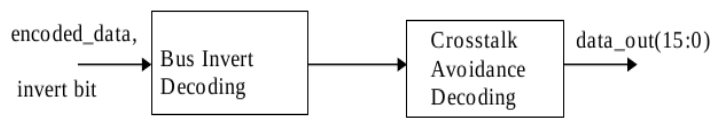

Figure 8: Block diagram for 16-bit Decoder Module 


\section{SimUlation RESUlts}

Simulation of various blocks was done using GHDL simulator[9]. Waveform viewer used was GTKWave. The simulation results for the encoder module is given in Figure 9 with data_in[15:0] is the input. The data_in[15:0] input is first CAC coded, then the bus invert coding is done for CAC coded output including the group complement bit. bus_out[25:0] and invert are the final outputs of the encoder module. By analyzing the simulation result, it can be concluded that the crosstalk avoidance property is maintained even after combining with bus invert coding which is performed to obtain low power.

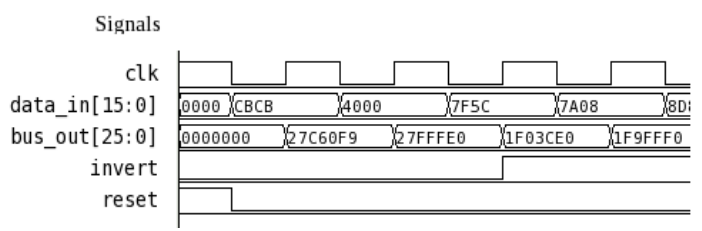

Figure 9: Simulation Results for Encoder Module

The simulation results for the decoder module is given in Figure10.The input to this integrated block is encoded_data[25:0] and invert, which are outputs of the module. The invert bit carries the information about the inversion of data. The output obtained after bus invert decoding is given to crosstalk avoidance decoder block. The decoded output denoted as data_out[15:0], which is same as that of the data given to the encoder module.

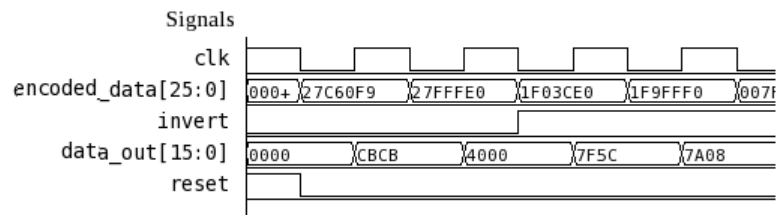

Figure 10: Simulation Results for Decoder Module

\section{CONCLUSIONS AND FUTURE WORK}

In this work, Forbidden Pattern Condition Codes (FPC) and Bus Invert method was used to address the cross talk and to reduce the power consumption respectively. Here, it was decided to place FPC (for crosstalk avoidance) as the first block to ensure that after performing bus invert coding(for low power) also, the crosstalk avoidance property will be maintained. If the coding scheme is reversed, that is first bus invert coding followed by crosstalk avoidance coding; then the criteria for low power consumption will not be met. The 16-bit coding scheme explained has the advantage that it can be extended to a higher bus width of 32 bits, 48 bits, 64 bits etc. By incorporating crosstalk avoidance and low power coding, the SoC reliability can be enhanced and the overall energy dissipation can be lowered.

In the next phase of this work, it is proposed to include error correction mechanisms also, which further improves the reliability of the system. But the error correction principles sholud be applied such that it will not alter the properties of forbidden pattern condition codes (which avoids crosstalk) and bus invert coding (which provides low-power).

\section{REFERENCES}

[1] Chunjie Duan, Anup Tirumala and Sunil P. Khatri, "Analysis and Avoidance of Cross- talk in On-Chip Buses", pages: 133-138, August 2001.

[2] Ahmed Elkammar, Norman Scheinberg and Srinivasa Vemuru, "Bus Encoding Scheme To Eiminate Unwanted Signal Transitions", Proceedings of the Third IEEE International Workshop on Electronic Design, Test and Applications (DELTA'06), IEEE 2005.

[3] Partha Pratim Pande, Haibo Zhu, Amlan Ganguly, Cristian Grecu, "Energy Reduction through Crosstalk Avoidance Coding in NoC Paradigm" Proceedings of the 9th EUROMICRO Conference on Digital System Design, Pages: 689-695, IEEE 2006

[4] Sudeep Pasricha and Nikil Dutt, "On-Chip Communication Architectures System on chip Interconnect ", Morgan Kaufmann Publishers, 2008 edition.

[5] Srinivasa R. Sridhara, Arshad Ahmed, and Naresh R. Shanbhag, "Area and Energy-Efficient Crosstalk Avoidance Codes for On-Chip Buses", Proceedings of the IEEE International Conference on Computer Design (ICCD'04), pages:12-17, October 2004.

[6] Mircea R. Stan, Member, IEEE, and Wayne P. Burleson, Member, IEEE, "Bus-Invert Coding for Low-Power I/O",IEEE Transactions on Very large Scale Integration (VLSI) Systems, Vol. 3, No. 1, March 1995.

[7] Rung-Bin Lin and Chi-Ming Tsai, Department of Computer Engineering and Science, Yuan-Ze University, "Theoretical Analysis of Bus-Invert Coding", Proceedings 43rd IEEE Midwest Symp. on Circuits and Systems,Lansing MI, 8-11, Aug 2000

[8] Chunjie Duan, Victor Cordero, Sunil P. Khatri, "Efficient On-Chip Crosstalk Avoidance CODEC Design" IEEE Transactions on Very large Scale Integration (VLSI) Systems, May 2009.

[9] http://ghdl.free.fr/ghdl/index.html

[10] http://www.vlsitechnology.org/html/sx_description.html

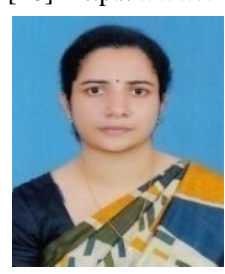

Anitta Thomas was born in Kerala, India. She took her B.Tech in Electronics and Communication Engineering from Mahatma Gandhi University College of Engineering, Thodupuzha(2004) and M.Tech from Model Engineering College, Thrikkakkara (2010).She is working as Assistant Professor at Viswajyothi College of Engineering and Technology, Vazhakulam for the last 11 years. Her fields of interest include VLSI and Embedded Systems. She is a life-time member of 'Indian Society for Technical Education' (Life Membership Card No: LM 100967). (E-mail: anitta.t@gmail.com)

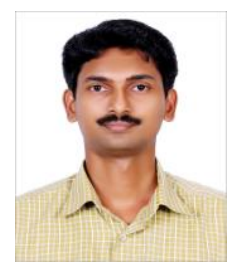

Shinoj J Vattakuzhi was born in Kerala, India. He took his B.Tech in Electronics Engineering from College Of Engineering, Chengannoor (2000). He is working as Sub Divisional Engineer in Bharath Sanchar Nigam Ltd, Ernakulam for the past 15 years. His fields of interest include VLSI, GSM and CDMA technology. (E-mail: shinojvattakuzhi@gmail.com) 